\title{
A setup for resonant inelastic soft $x$-ray scattering on liquids at free electron laser light sources
}

\author{
Kristjan Kunnus, ${ }^{1,2, a)}$ Ivan Rajkovic, ${ }^{3}$ Simon Schreck, ${ }^{1,2}$ Wilson Quevedo, ${ }^{3}$, b) Sebastian Eckert, ${ }^{1}$ Martin Beye, ${ }^{1}$ \\ Edlira Suljoti, ${ }^{1}$ Christian Weniger, ${ }^{1}$ Christian Kalus, ${ }^{4}$ Sebastian Grübel, ${ }^{3, c}$ ) Mirko Scholz, ${ }^{3}$ Dennis Nordlund, ${ }^{5}$ \\ Wenkai Zhang, ${ }^{6}$ Robert W. Hartsock, ${ }^{6}$ Kelly J. Gaffney, ${ }^{6}$ William F. Schlotter, ${ }^{7}$ Joshua J. Turner, ${ }^{7}$ \\ Brian Kennedy, ${ }^{8}$ Franz Hennies, ${ }^{8}$ Simone Techert,, 3 , 9) d) Philippe Wernet, ${ }^{1, \mathrm{e})}$ and Alexander Föhlisch ${ }^{1,2, \mathrm{f})}$ \\ ${ }^{1)}$ Institute for Methods and Instrumentation for Synchrotron Radiation Research, Helmholtz-Zentrum Berlin GmbH, \\ Albert-Einstein-Str. 15, 12489 Berlin, Germany \\ ${ }^{2)}$ Institut für Physik und Astronomie, Universität Potsdam, Karl-Liebknecht-Straße 24/25, 14476 Potsdam, \\ Germany \\ ${ }^{3)}$ IFG Structural Dynamics of (Bio)chemical Systems, Max Planck Institute for Biophysical Chemistry, \\ Am Faßberg 11, 37070 Göttingen, Germany \\ 4) Abteilung Betrieb Beschleuniger BESSYII, Helmholtz-Zentrum Berlin GmbH, Albert-Einstein-Str. 15, \\ 12489 Berlin, Germany \\ 5) Stanford Synchrotron Radiation Lightsource, SLAC National Accelerator Laboratory, Menlo Park, \\ CA 94025 USA \\ ${ }^{6)}$ PULSE Institute, SLAC National Accelerator Laboratory, Menlo Park, CA 94025 USA \\ ${ }^{7)}$ Linac Coherent Light Source, SLAC National Accelerator Laboratory, Menlo Park, \\ $C A 94025$ USA \\ ${ }^{8)} M A X-l a b, P O$ Box 118, 221 00 Lund, Sweden \\ 9) Advanced Study Group of the MPG, CFEL, Notkestraße 85, 22853 Hamburg, \\ Germany
}

(Dated: October 24, 2012)

We present a flexible and compact experimental setup that combines an in vacuum liquid jet with a $\mathrm{x}$-ray emission spectrometer to enable static and femtosecond time-resolved resonant inelastic soft x-ray scattering (RIXS) measurements from liquids at free electron laser (FEL) light sources. We demostrate the feasibility of this type of experiments with the measurments performed at the Linac Coherent Light Source FEL facility. At the FEL we observed changes in the RIXS spectra at high peak fluences which currently sets a limit to maximum attainable count rate at FELs. The setup presented here opens up new possibilities to study the structure and dynamics in liquids.

\section{INTRODUCTION}

X-ray emission spectroscopy (XES) and resonant inelastic $\mathrm{x}$-ray scattering (RIXS) probe the valence electronic structure in an atom specific and chemical state selective manner. ${ }^{1,2}$ The cross section of a RIXS process is defined by the projection of valence molecular orbitals on the localized atomic core orbitals. In combination with stringent symmetry selection rules RIXS measurements provide the experimental analogue of the theoretical decomposition of molecular orbitals to a linear combination of atomic orbitals. ${ }^{1,3}$ In addition, nuclear and electronic wavepacket dynamics on the intrinsic femtosecond time scale of core excited species can be clocked. ${ }^{4-6}$ These experiments rely on the high average brilliance of

\footnotetext{
a) kristjan.kunnus@helmholtz-berlin.de

b) Current address: Institute for Methods and Instrumentation for Synchrotron Radiation Research, Helmholtz-Zentrum Berlin GmbH, Albert-Einstein-Str. 15, 12489 Berlin, Germany

c) Current address: Paul Scherrer Institut, 5232 Villingen PSI, Switzerland

d) stecher@gwdg.de

e) wernet@helmholtz-berlin.de

f)alexander.foehlisch@helmholtz-berlin.de
}

modern third generation synchrotron radiation sources. With the advent of femtosecond x-ray pulses from soft xray FELs, XES and RIXS can be brought into the time domain to excite and follow molecular and chemical dynamics in a pump-probe approach. ${ }^{7,8}$ In particular, when it comes to following molecular dynamics in solution with time-resolved x-ray spectroscopic techniques, the photon-in and photon-out based method of time-resolved RIXS (tr-RIXS) in the soft x-ray range uniquely complements femtosecond time-resolved soft x-ray absorption spectroscopy (tr-XAS). ${ }^{9-15}$ The potential of femtosecond tr-RIXS has been recently demonstrated by the experimental determination of the high density and low density liquid phases of silicon ${ }^{16}$, which stands for the transient phases of tetragonally bound solids, including the phase diagram of ice and water.

In this work we report on the properties of a dedicated experimental station for femtosecond time resolved XES and RIXS for liquid phase molecular dynamics and chemistry. The design of the setup is based on the experience of previous realizations of steady-state soft $\mathrm{x}$-ray RIXS studies of liquids. ${ }^{17-20}$ It can be utilized as a transportable and flexible instrument both at synchrotron radiation sources and at the soft x-ray beamlines of the LCLS at the SLAC National Accelerator Laboratory ${ }^{21}$ or other FEL facilities. 
In a first step we describe and characterize thoroughly the science and FEL-technology driven design parameters of the setup (Section II). In a second step we show how the experimental results on identical samples compare for the exposure to synchrotron and FEL radiation (Section III). In particular, we measured RIXS spectra of typical solvents (methanol and ethanol) and a solute $\left(\mathrm{Fe}(\mathrm{CO})_{5}\right.$ at $1 \mathrm{~mol} / \mathrm{l}$ concentration in ethanol) and we compare in detail the spectra and parameters of the different light sources. With this we show that over a wide range of FEL radiation parameters the short duration, high brilliance pulses of the FEL can be utilized to obtain equivalent spectral information to the synchrotron. These measurements demonstrate the experimental methodology and feasibility of femtosecond tr-RIXS in liquid systems.

\section{THE EXPERIMENTAL SETUP}

The experimental setup is depicted schematically in Figure 1 and in greater technical detail in Figure 2. The main components are an in vacuum liquid jet and a RIXS spectrometer consisting of a slit, gratings and a detector. Details of both are discussed below. Differential pumping stages with elongated pin holes are used to decouple the beamline vacuum (Figure 2, typical pressure $\mathrm{p}_{1}=10^{-8}$ mbar) from the pressure in the main chamber (typically $\mathrm{p}_{2}=10^{-3} \mathrm{mbar}$ ). A thin x-ray transparent $\mathrm{C}$ parylene foil is used to decouple the detector environment from the main chamber giving a typical detector pressure of $\mathrm{p}_{3}=10^{-8}-10^{-6}$ mbar. A GaAsP diode can be mounted to find spatial overlap between the x-ray beam and the liquid jet and to record total fluorescence yield $\mathrm{x}$-ray absorption spectra. Tools to find spatial and temporal overlap of optical laser and x-ray beams in tr-RIXS experiment can be easily mounted. These are described in detail in Ref. 22.

A similar experimental chamber with a liquid jet has already been used at the FLASH FEL facility in Hamburg for the investigation of the interaction of FEL radiation with water and solid samples in combination with ultrafast x-ray scattering and optical/UV spectroscopy techniques. ${ }^{23-25}$

\section{A. The liquid jet: sample delivery}

One key part of the experimental station presented here is a sample delivery system that replaces the material in the interaction volume for each x-ray burst. This guarantees that consecutive x-ray pulses do not probe sample molecules that are damaged by previous x-ray probe or optical pump pulses. This requirement is met here with in vacuum liquid jet technology. ${ }^{26-28}$

The liquid jet nozzles (purchased from Microliquids $\mathrm{GmbH}$ ) are specially designed for the liquid to run in the laminar flow regime. ${ }^{26}$ The length of this laminar flow re-

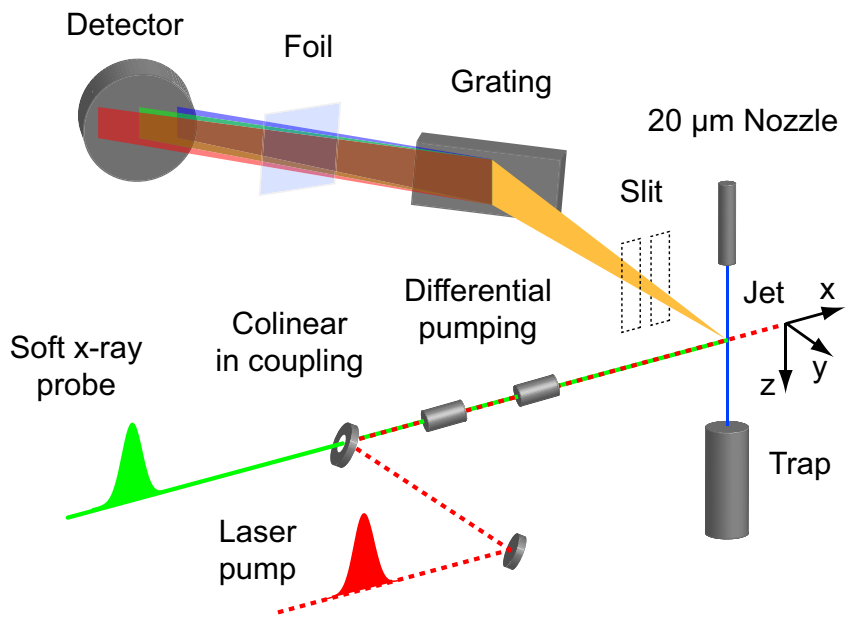

Figure 1. Schematic depiction of the experimental setup. The RIXS spectrometer is a modified XES 350 with three gratings (only one is drawn here). A MCP detector is operated behind a vacuum separating foil. A differential pumping stage with tubes serving as pin holes is used to decouple the vacuum in the experimental chamber from the beamline vacuum. The coordinate system defines the geometry with $\mathrm{x}$ as the propagation direction of the x-ray beam, $y$ as the optical axis of the RIXS spectrometer and $\mathrm{z}$ the propagation direction of the liquid jet. For collinear incoupling of an optical laser we use a setup provided as part of the SXR beamline at LCLS ${ }^{21}$.

gion in vacuum is usually on the order of a few millimeters before the jet starts to break up to form droplets. The xray beam crosses the jet in the laminar flow region. The diameter of the liquid jet in the laminar flow region is well defined and of a similar size as the inner diameter of the nozzle, which was $20 \mu \mathrm{m}$ for the experiments presented here. The liquid is frozen in a cold trap once it passes the interaction region (Figure 2).

Typical flow rates are in the range of $1-2 \mathrm{ml} / \mathrm{min}$. At these flow rates, assuming a $20 \mu \mathrm{m}$ jet, the sample in a $100 \mu \mathrm{m}$ high interaction region is replaced at a rate of $0.5-1 \mathrm{MHz}$. This ensures that each FEL pulse hits a fresh volume of the sample (typical warm linac based FELs operate around $100 \mathrm{~Hz}$ repetition rate (LCLS up to $120 \mathrm{~Hz}$ ), while superconducting linacs reach around $1 \mathrm{MHz}$ repetition rates (FLASH, XFEL)). At BESSYII, with a $500 \mathrm{MHz}$ repetition rate, this is not the case, but the sample is still continuously replenished, minimizing radiation damage and avoiding thermal degradation processes which take place on time scales longer than a millisecond.

For pumping the liquid sample into the vacuum, a commercial HPLC (high-performance liquid chromatography) pump system from Jasco Inc. was used. The pump system consists of a degasser, a mixer and a pump (Figure 2). The liquid sample flows first into the degasser where the gaseous components are removed. Degassing the liquid is important to ensure stable flow of the jet. Following the degasser, the liquid arrives to the 3-channel mixer unit which allows easily to mix and switch be- 

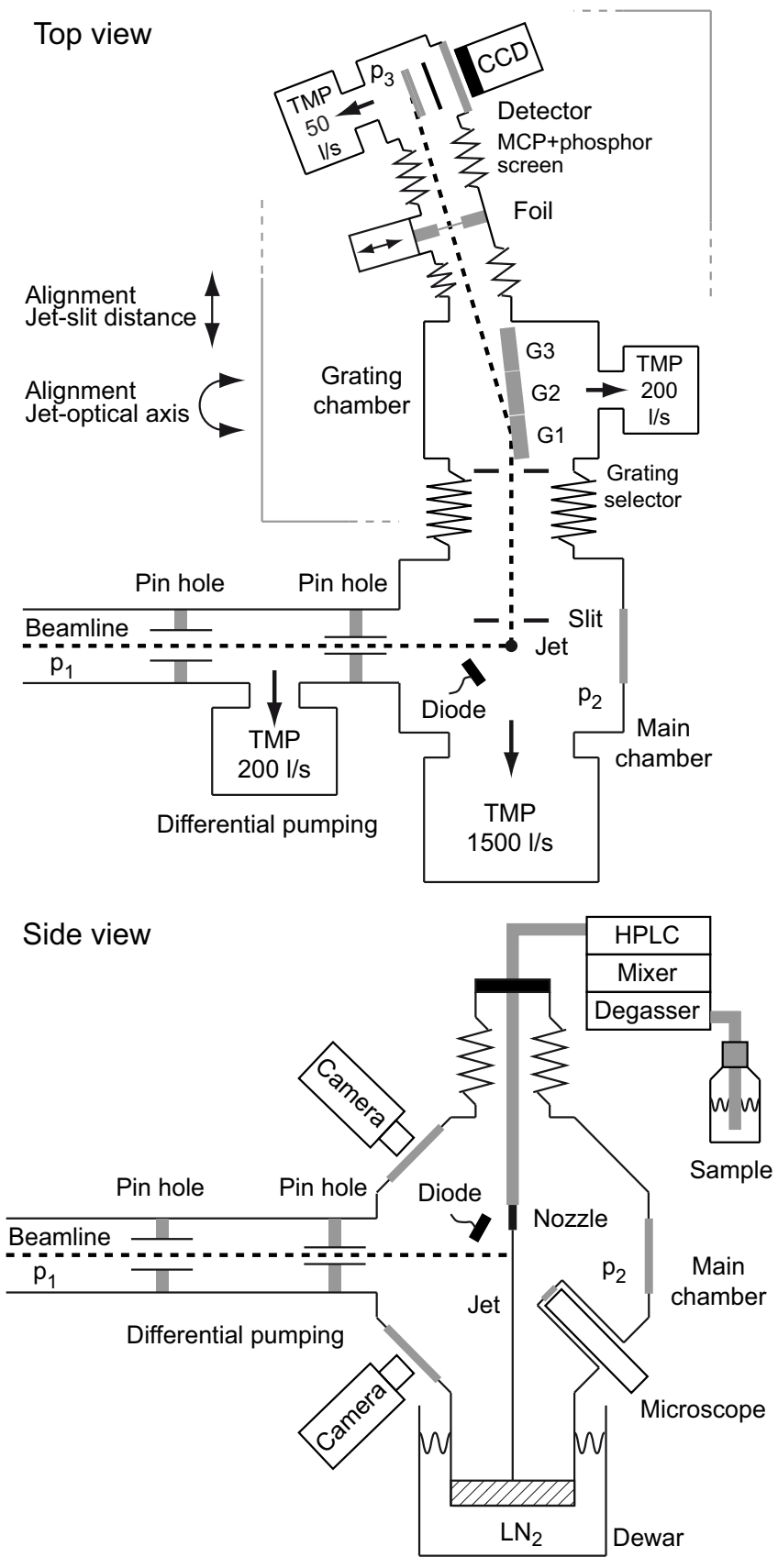

Figure 2. The components of the experimental setup: top view (upper panel), side view (lower panel).

tween up to three different liquids without interrupting the flow of the jet. The sample then runs through the HPLC pump unit which pumps the sample under pressure of 10-100 bar into the vacuum of the experimental chamber.

\section{B. The RIXS spectrometer}

The optical layout of our RIXS spectrometer is the Graze spectrometer XES $350^{29}$, which is a grazing-
Table I. Photon energy ranges reachable with the spectrometer. In the parenthesis is the optimal resolution of the spectometer at the given energy for $20 \mu \mathrm{m}$ source width.

\begin{tabular}{cccc}
\hline \hline & order & $E_{\min }(\mathrm{eV})$ & $E_{\max }(\mathrm{eV})$ \\
\hline G1 & 1. & $240(0.15)$ & $425(0.49)$ \\
& 2. & $480(0.31)$ & $850(0.97)$ \\
G2 & 1. & $100(0.08)$ & $450(1.63)$ \\
& 2. & $400(0.65)$ & $900(3.27)$ \\
G3 & 1. & $50(0.04)$ & $200(0.72)$ \\
& 2. & $125(0.14)$ & $375(1.26)$ \\
\hline \hline
\end{tabular}

incidence spherical concave grating spectrometer with Rowland geometry mounting. The spectrometer is equipped with an entrance slit where the size can be continuously varied between $400 \mu \mathrm{m}$ and $0 \mu \mathrm{m}$ (Figure 1 and 2). As discussed in detail below, the dimensions of the liquid jet create a geometrically well defined source that allows the spectrometer to operate with a fully opened slit in "slitless mode" to enhance the transmission of the spectrometer without deteriorating the resolution.

The spectrometer houses three different gratings which can be selected by adjusting the position of the grating selector baffles in front of the gratings (Figure 2). In Figure 3 we show the efficiency of the gratings over a wide photon energy range. Table I lists the energy ranges and energy resolutions reachable with the current spectrometer taking into account also the mechanical constraints in positioning of the detector. The spectrometer is mounted in a $90^{\circ}$ scattering geometry with respect to the incident $\mathrm{x}$-ray beam (Figure 1).

\section{The detection scheme}

To record RIXS spectra, the spectrometer is equipped with a detector unit that consists of a MCP stack, a phosphor screen and a CCD. The detector unit has single photon sensitivity, multihit capablity and it can be read out for each x-ray burst at an FEL source. This effort is necessary to avoid averaging RIXS spectral features that depend upon the statistically varying SASE (self amplified spontaneous emission) x-ray pulses. By avoiding averaging, we can sort and bin the data based on the values of key parameters (for example with respect to incident intensity or with respect to pump-probe delay in tr-RIXS experiments).

Due to the high peak flux of FELs, an important aspect necessary to consider is the linear range of the detector used in the experiment. At the SXR beamline of LCLS, the unmonochromatized beam at $540 \mathrm{eV}$ delivers about $8 \cdot 10^{12}$ photons per pulse to the experiment. Using ethanol in the jet resulted on average in 330 counts per pulse at our detector (shot by shot readout was possible at LCLS due to the repetition rate of $60 \mathrm{~Hz}$ ). This is 
at the limit of the single photon counting regime for our detector where the individual counts are still distinguishable and not merged. Due to monochromatization and attenuation the count rates of the actual measurements at LCLS were well in the linear regime, typically in range of $0.1-10$ counts per pulse. Because of the possibility of more than one count per pulse, the multi hit capability of the detector becomes essential. For this reason a CCD detector was employed and not a resistive anode or a delay line detector commonly used with these type of spectrometers at synchrotrons. For the BESSYII experiments the same detector arrangement is used. There, the CCD camera is read out at $15 \mathrm{~Hz}$ which always results in less than 100 counts per frame.

\section{The efficiency of the system}

In order to determine the feasibility and data acquisition time for a planned experiment, we need to consider the total efficiency of the setup. The product of the grating efficiency and the transmission of the foil are plotted in Figure 3. With the acceptance of $10^{-5}$ for isotropic radiation of the source ${ }^{29}$, with the transmission of the gratings and foil of $10 \%$ and with the $10 \%$ detector efficiency $^{29,30}$, the overall efficiency of the spectrometer is on the order of $10^{-7}$.

\section{E. The slitless mode of operation}

Due to the low cross section for soft $\mathrm{x}$-ray RIXS it is crucial to maximize the efficiency of the spectrometer. With a sub-100 $\mu \mathrm{m}$ jet as the sample we can increase the acceptance of the spectrometer by opening the entrance slit (slitless mode of operation). This mode of operation is characterized below.

The liquid jet runs vertically from the nozzle above into a cryogenic trap below as depicted in Figure 1. The RIXS spectrometer is mounted in the relative orientation where the jet is parallel to the lines of the grating (jet is parallel to the sagittal plane, Figure 1). The vertically elongated source volume is defined by the overlap of the liquid jet and the x-ray beam. We now exemplify the advantages of the slitless operation for this experimental arrangement using grating G1. The acceptance angles for grating G1 at a jet-slit distance of $10 \mathrm{~mm}$ are $0.5^{\circ}$ and $8.1^{\circ}$ in the meridional and sagittal plane, respectively. The typical width of the source is $20 \mu \mathrm{m}$ (jet diameter) and typical height of the source is $100 \mu \mathrm{m}$ (vertical synchrotron beam focus size with beamline slit set to $100 \mu \mathrm{m}$ ). It follows that at the spectrometer entrance slit the width (height) of the accepted x-ray emission beam is $105 \mu \mathrm{m}(1520 \mu \mathrm{m})$. In order not to lose any light at the entrance slit (Figure 1), the slit thus has to be opened wider than $105 \mu \mathrm{m}$. This defines the slitless mode of operation. Closing the slit down to $20 \mu \mathrm{m}$ decreases the count rate by a factor of 5 without any gain in resolution.

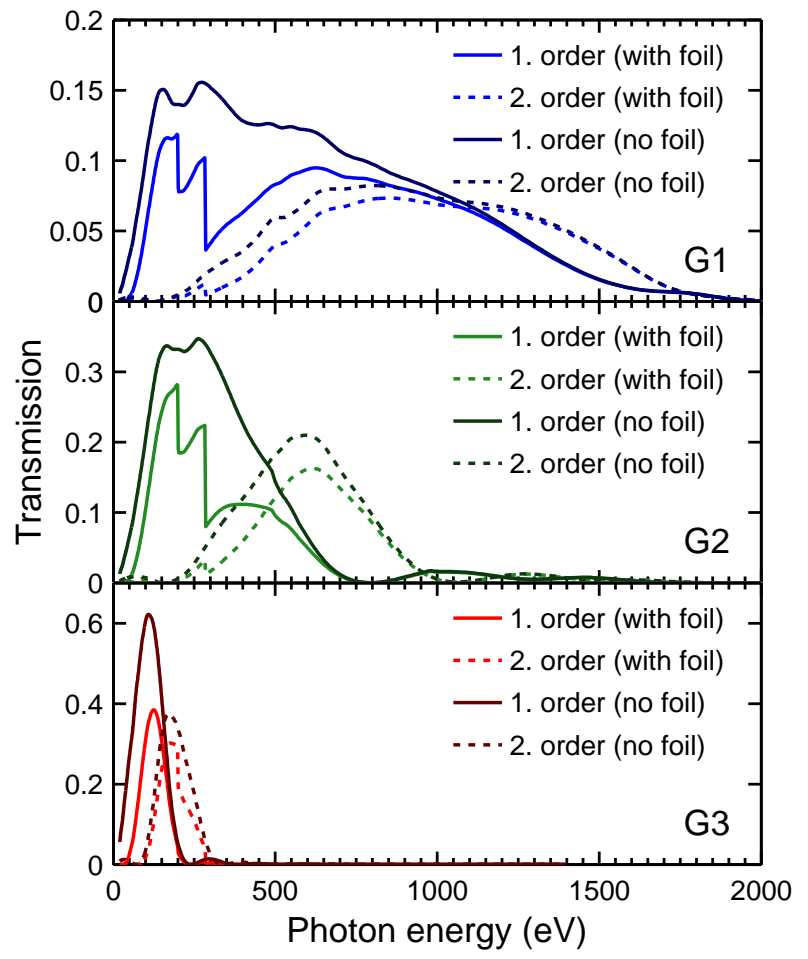

Figure 3. Calculated photon energy dependence of the combined transmission of the gratings and the C-parylene foil (thickness $234.5 \mathrm{~nm}$ ). Grating efficiencies were calculated using the REFLEC program ${ }^{31-33}$. Data for the transmission of the foil were taken from Ref. 34. Darker curves show transmission without the foil (only grating efficiency).

At the LCLS SXR beamline, we could further utilize the bendable mirror refocusing optics, and create an elongated focal spot along the liquid jet. This allowed us to reduce the incident fluence without decreasing the photon flux. This is possible because the acceptable source length of the RIXS spectrometer in this direction (z-axis in Figure 1) is several millimetres. The control of the incident $\mathrm{x}$-ray fluence is very important as the power of the FEL $\mathrm{x}$-ray burst must stay below the radiation damage threshold to study properties that are not influenced by the x-rays (discussed in Section III).

\section{F. Sensitivity to source position and stability}

In the slitless mode, the liquid jet is displaced from its ideal position on the Rowland circle due to the distance between the spectrometer entrance slit and the actual liquid jet position. We need to compensate this deviation by repositioning the detector.

Figure 4 depicts displacements of the source along the $\mathrm{x}$-axis (along the $\mathrm{x}$-ray beam, Figure 1 ) and along the $\mathrm{y}$ axis (perpendicular to the $\mathrm{x}$-ray beam and along the optical axis of the spectrometer). According to equation 22 in Ref. 35, the best position of the detector (meridional 


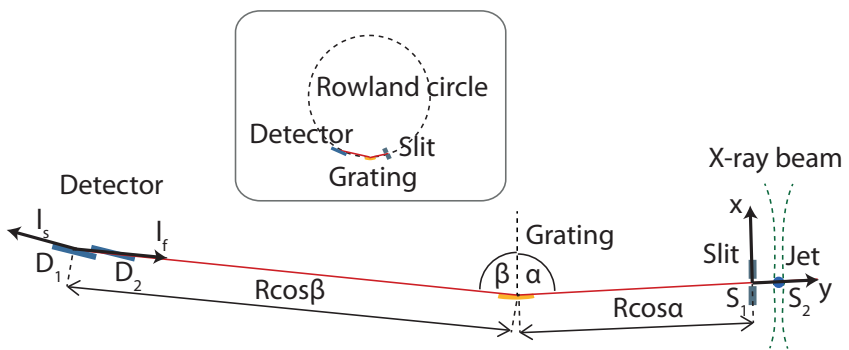

Figure 4. Schematic geometry of the spectrometer in the meridional plane. The detector position $\mathrm{D}_{1}$ (on the Rowland circle) corresponds to a source position $\mathrm{S}_{1}$ (entrance slit, on the Rowland circle) and detector position $\mathrm{D}_{2}$ (off the Rowland circle) corresponds to a source position $\mathrm{S}_{2}$ (jet, off the Rowland circle). Displacements of the jet from the entrance slit along the optical axis $(y)$ correspond to displacements of the diffracted beam focus position with respect to the Rowland circle $\left(l_{f}\right)$. Note that for the jet in the middle of the entrance slit we have $y=0$ and $l_{f}=0$. Also misalignments of the jet position perpendicular to the optical axis $(x)$ can occur. Movements of the jet along the $\mathrm{x}$-direction shift the spectrum on the detector (set tangential to the Rowland circle) by $l_{s}$. The inset shows the full Rowland circle and positions of the entrance slit, the grating and the detector.

focus) is found if the detector is positioned at a distance $r^{\prime}$ from the grating:

$$
r^{\prime}=\cos ^{2} \beta\left(\frac{\cos \alpha+\cos \beta}{R}-\frac{\cos ^{2} \alpha}{r}\right)^{-1}
$$

where $\alpha$ and $\beta$ are the angle of incidence and diffraction, respectively. The conventional definitions for the angles $\alpha$ and $\beta$ are used (Figure 4). $R$ is the curvature radius of the grating and $r=R \cos \alpha+y$ is the distance between the grating and the source with $y$ being the distance between the Rowland circle and the source (along the line connecting the centre of the grating and the source, see Figure 1 and 4 ). The Rowland geometry is a special case of equation 1 where $r=R \cos \alpha$ and $r^{\prime}=R \cos \beta$.

Figure 5 depicts the measured and calculated results for the relationship between resolution and count rate and the displacements of the liquid jet. At the BESSYII U41-PGM beamline, we measured the oxygen K-emission spectrum of pure ethanol. Grating G1 was used in second order. The entrance slit of the spectrometer was fully open $(\sim 400 \mu \mathrm{m})$ and the incident photon energy was set to $550 \mathrm{eV}$.

Figure 5a shows how the position of the image focus depends on the displacements of the jet if the jet is moved along the $\mathrm{y}$-axis. The position of the focus is defined as $l_{f}=R \cos \beta-r^{\prime}$ (see also Figure 4). For each $y$-position of the jet, the optimal focus was found by moving the detector along the ray of emitted radiation and observing the resolution of the emission spectrum. We found an approximately linear relationship in agreement with equation 1. The count rate is unaffected by this displacement (not shown). The displacement of the jet with
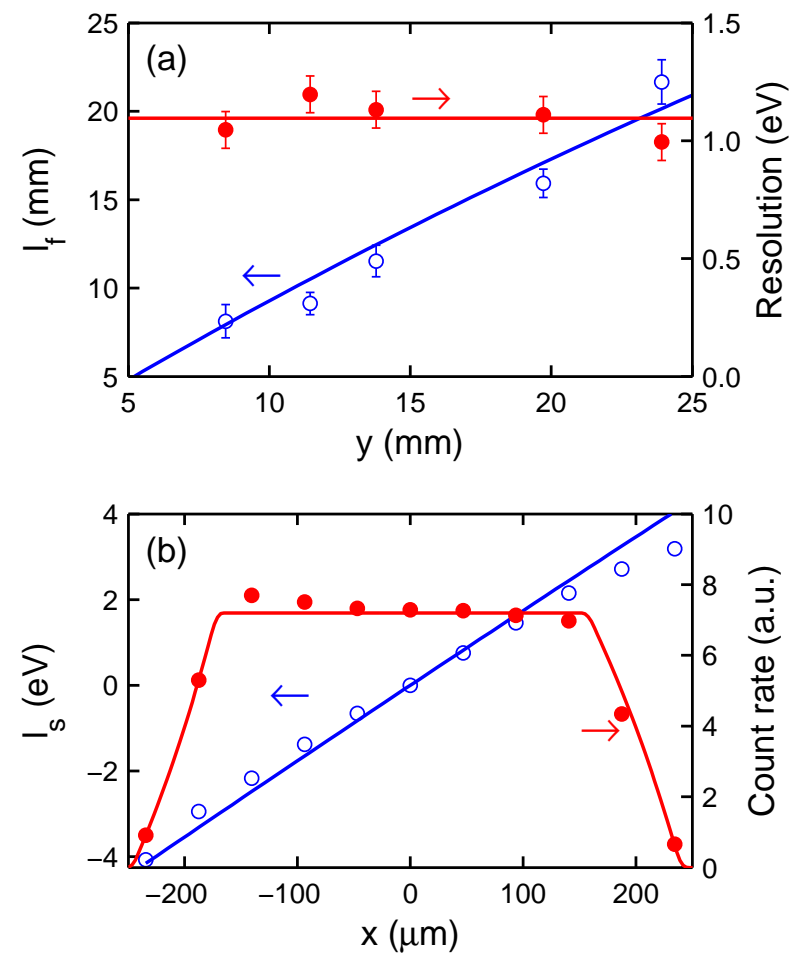

Figure 5. Response of the RIXS spectrometer to displacements of the liquid jet in the slitless mode of operation (measurement: markers; simulation: lines). Measurements were done at the $\mathrm{O}$ K-edge (main emission line at $525 \mathrm{eV}$ ) using grating G1 in 2nd order with a diameter of the jet of $20 \mu \mathrm{m}$. (a) Shift of the focus position $\left(l_{f}\right.$, blue) and resolution (red) for jet displacements along y-axis. The blue empty circles are measured focus positions and the blue line shows calculations based on equation 1. (b) Shift of the spectrum $\left(l_{s}\right.$, blue) and count rate (red) for jet displacements along x-axis. The blue empty circles are measured shifts of the spectrum and the blue line corresponds to a calculation based on the grating equation. Experimental count rates (red filled circles) are compared to the calculated grating illumination for grating G1 at an entrance slit width of $400 \mu \mathrm{m}(20 \mu \mathrm{m}$ jet, offset from the slit $10 \mathrm{~mm}$ ).

respect to the Rowland circle thus needs to be compensated by an approximately equal amount in the detector displacement in order to keep the resolution constant.

The general condition given by equation 1 does not minimize the optical aberrations as well as the Rowland circle condition. ${ }^{35,36}$ By displacing the source away from the Rowland circle one increases the magnitude of aberrations and a question arises whether this affects the resolution. Our results in Figure 5a show however that in the range of distances up to $25 \mathrm{~mm}$ between the jet and the entrance slit, the effect on the resolution is negligible. The insensitivity of the resolution to the jet position reflects the dominant role of the $20 \mu \mathrm{m}$ source width on the spectrometer resolution and the small displacement from the Rowland circle relative to the distance between the grating and the slit: $y /(R \cos \alpha)<1 \%$. The count 


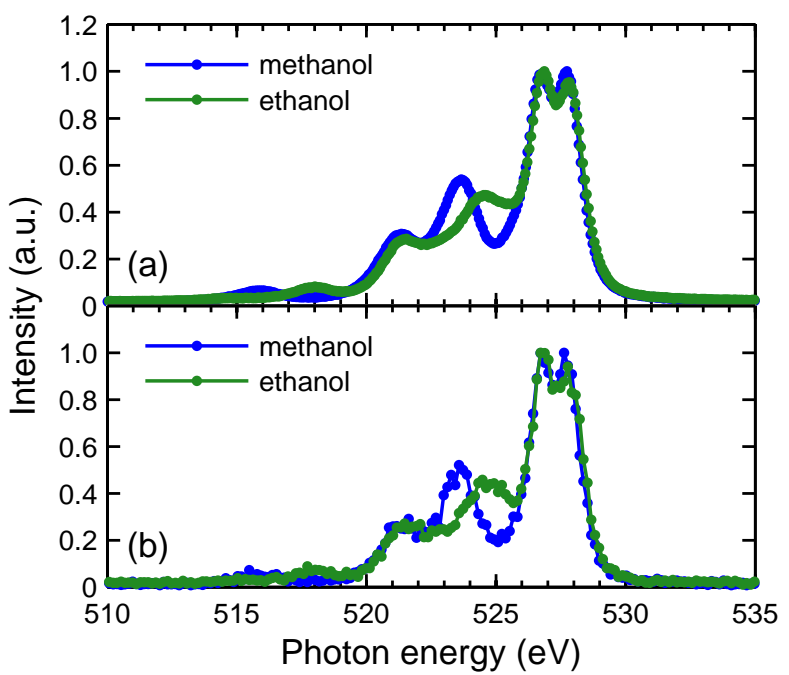

Figure 6. Comparison of liquid methanol and ethanol RIXS spectra taken at an incident photon energy of $540 \mathrm{eV}$ and measured (a) at the BESSYII U41-PGM beamline and (b) at the LCLS SXR beamline. For details on the experimental parameters see Table II.

rate and resolution are thus clearly unaffected by $\mathrm{mm}$ movements of the jet along the optical axis of the spectrometer (y-axis, Figure 1 and 4).

The situation is different when the jet is moved along the x-ray beam (along the x-axis, Figure 1 and 4) as the results in Figure 5b show. Then the measurement in slitless mode is very sensitive to movements of the jet. These movements change the angle of incidence on the grating. According to the grating equation this directly translates to shifts in the measured spectra. We recorded x-ray emission spectra for several $x$-positions of the jet. We found that jet movements on the order of $100 \mu \mathrm{m}$ change the apparent energy of spectral lines on the detector by $2 \mathrm{eV}$ (Figure $5 \mathrm{~b}$ ). The count rate stays constant over the range of $\sim 400 \mu \mathrm{m}$ which corresponds to the size of the fully opened entrance slit. It follows that it is very important to control the position of the jet. In addition, one needs to apply great caution when comparing spectra measured at different relative alignments of jet and spectrometer. We note that during the experiments, we typically observe no drifts or vibrations of the liquid jet in the order of approximately $1 / 4$ of the jet diameter (resolution of the microscope looking at the jet).

\section{RESULTS}

In Figure 6 we show how RIXS spectra of the solvents ethanol and methanol measured at BESSYII and at LCLS compare. The spectra were recorded at an incident photon energy of $540 \mathrm{eV}$ in the broad main-edge feature of the x-ray absorption spectra with an excitation bandwidth of $3 \mathrm{eV}$. We observe for both ethanol

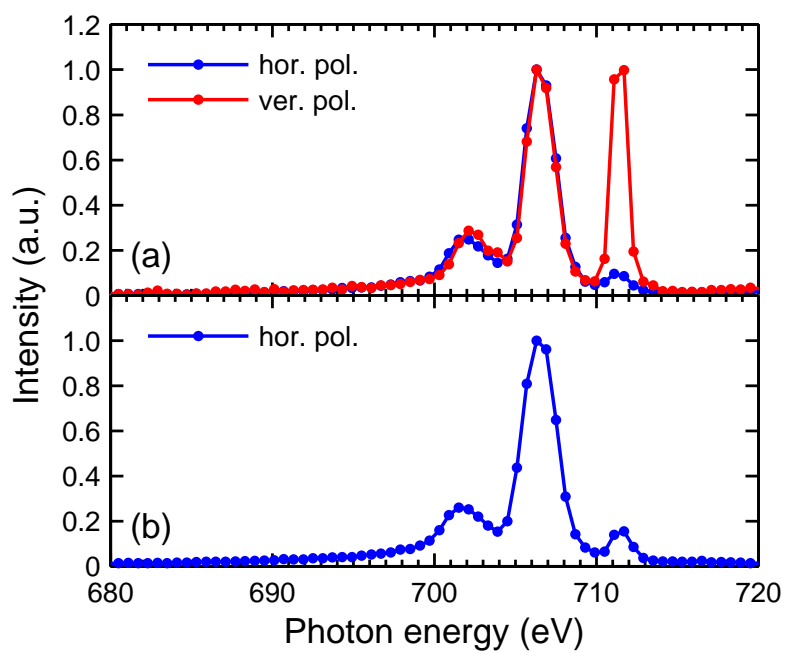

Figure 7. Comparison of $\mathrm{Fe}(\mathrm{CO})_{5}$ RIXS spectra taken at an incident photon energy of $711.5 \mathrm{eV}$ and measured (a) at the BESSYII UE56/1-PGM beamline and (b) at the LCLS SXR beamline. The BESSYII spectra in (a) were measured with horizontally and vertically polarized x-rays. The LCLS spectra we measured with horizontally polarized incident x-rays. For details on the experimental parameters see Table III.

and methanol the typical splitting of the highest energy emission peak. ${ }^{37,38}$ The spectra measured at LCLS (Figure 6b) compare well to the results from BESSYII (Figure $6 \mathrm{a})$, despite peak fluence of 1.0 photons $/\left(\mathrm{ps} \times \mu \mathrm{m}^{2}\right)$ at the synchrotron and $1.3 \cdot 10^{6}$ photons $/\left(\mathrm{ps} \times \mu \mathrm{m}^{2}\right)$ at LCLS. In Figure 6 the accumulation time of the BESSYII spectra was $30 \mathrm{~min}(3600 \mathrm{cts} / \mathrm{s})$ and the accumulation time of the LCLS spectra was $40 \mathrm{~min}(7 \mathrm{cts} / \mathrm{s})$. For a detailed comparison of incident flux, photons per pulse, repetition rate, pulse length, focus spot size, peak fluence and count rates in the methanol experiments see Table II. For peak fluences exceeding $8 \cdot 10^{6}$ photons $/\left(\mathrm{ps} \times \mu \mathrm{m}^{2}\right)$ at LCLS we detected considerable changes of the spectrum, which we took as an upper threshold for our measurements. Similar threshold fluences were observed for ethanol and methanol.

In Figure 7, we show the $\mathrm{Fe}_{3}$-edge RIXS spectra measured at BESSYII and at LCLS from $\mathrm{Fe}(\mathrm{CO})_{5}$ with a concentration of $1 \mathrm{~mol} / \mathrm{l}$ in ethanol. The parameters for this measurement are summarized in Table III. The RIXS spectra were measured at an incident photon energy of $711.5 \mathrm{eV}$ which corresponds to the main absorption peak of $\mathrm{Fe}(\mathrm{CO})_{5}$ at the iron $\mathrm{L}_{3}$ edge. Figure $7 \mathrm{a}$ depicts two spectra measured with different polarization at a beamline with variable polarization at BESSYII. Inelastic RIXS features remain unchanged while the intensity of the elastic peak changes in accordance with the $\cos ^{2} \theta$ law for elastic scattering $(\theta$ is the angle between the polarization plane of the incident radiation and the detection direction of the scattering radiation).

For peak fluences exceeding $5 \cdot 10^{7}$ photons $/\left(\mathrm{ps} \times \mu \mathrm{m}^{2}\right)$ at LCLS we detected considerable changes in the Fe $\mathrm{L}_{3}$ 
Table II. Relevant experimental parameters for the measurements of liquid methanol RIXS spectra at the LCLS SXR beamline and at the BESSYII U41-PGM beamline. RIXS spectra are displayed in Figure 6 . The incident photon energy was $540 \mathrm{eV}$ with a $3 \mathrm{eV}$ bandwidth (FWHM).

\begin{tabular}{lcc}
\hline \hline & LCLS & BESSYII \\
\hline Average incident flux $(1 / \mathrm{s})$ & $7.3 \cdot 10^{10}$ & $2.1 \cdot 10^{13}$ \\
Photons per pulse & $1.2 \cdot 10^{9}$ & $4.2 \cdot 10^{4}$ \\
Attenuation factor ${ }^{\mathrm{a}}$ & 20 & - \\
Repetition rate $(\mathrm{Hz})$ & 60 & $5 \cdot 10^{8}$ \\
Pulse length $(\mathrm{ps})$ & 0.16 & 60 \\
Spot size on the jet $(\mu \mathrm{m} \times \mu \mathrm{m})$ & $20 \times 300$ & $20 \times 40$ \\
Fluence per pulse $\left(\mathrm{mJ} / \mathrm{cm}^{2}\right)$ & 1.8 & $5.2 \cdot 10^{-4}$ \\
Peak fluence $\left(1 /\left(\mathrm{ps} \times \mu \mathrm{m}^{2}\right)\right)$ & $1.3 \cdot 10^{6}$ & 1.0 \\
Measured count rate $(1 / \mathrm{s})$ & 7 & 3600 \\
Estimated count rate $(1 / \mathrm{s})^{\mathrm{b}}$ & 13 & - \\
\hline \hline
\end{tabular}

a The attenuation factor is given with respect to an unattenuated but monochromatized beam. Compared to the unmonochromatized beam the attenuation is $\sim 200$.

b The estimated LCLS count rate is calculated from the measured count rate at BESSYII taking into account the different average incident flux. The discrepancy between measured and estimated LCLS count rate reflects the uncertainties in the average incident photon fluxes of LCLS and BESSYII.

edge RIXS spectrum of the $\mathrm{Fe}(\mathrm{CO})_{5}$ solution. For this reason the FEL beam intensity was decreased during the experiment using the gas attenuator installed in front of the beamline. It is clear from the comparison of spectra in Figure 7 that the RIXS spectrum measured at LCLS reproduces the spectrum measured at BESSYII without any distortions although the incident peak fluence was $10^{10}$ times larger at LCLS (Table III). In Figure 7 the accumulation time of the vertical polarization BESSYII spectrum was $15 \mathrm{~min}$ (10 cts/s) and the accumulation time of the horizontal polarization LCLS spectra was $4 \mathrm{~min}$ (480 cts/s).

We compare now the observed threshold peak fluence values for pure methanol/ethanol and for $\mathrm{Fe}(\mathrm{CO})_{5}$ ethanol solution. Distortions in the oxygen $\mathrm{O} \mathrm{K}$-edge RIXS spectra from pure methanol/ethanol were present if peak fluences of $8 \cdot 10^{6}$ photons $/\left(\mathrm{ps} \times \mu \mathrm{m}^{2}\right)$ were reached (540 eV incident photon energy) and distortions in the $\mathrm{Fe} \mathrm{L}_{3}$-edge RIXS spectra from $\mathrm{Fe}(\mathrm{CO})_{5}$ solution were found starting at peak fluences 5 . $10^{7}$ photons $/\left(\mathrm{ps} \times \mu \mathrm{m}^{2}\right)$. Therefore the threshold peak fluence for the $\mathrm{Fe}(\mathrm{CO})_{5}$ solution is $\sim 6$ times higher than for the pure methanol/ethanol.

Spectral distortions present in the RIXS spectra at high fluences are due to scattering of incident photons with already (valence) excited and/or distorted molecules created from interaction of photons in the beginning of the x-ray pulse. For a complete understanding of the observed fluence effects calculations taking into account details of the electronic structure and all relaxations chan-
Table III. Relevant experimental parameters for the experiment on the $\mathrm{Fe}(\mathrm{CO})_{5}$ solution at the LCLS SXR beamline and at the BESSYII UE56/1-PGM beamline. RIXS spectra are displayed in Figure 7. The photon energy was $711.5 \mathrm{eV}$ with an $0.5 \mathrm{eV}$ bandwidth at the LCLS and $0.3 \mathrm{eV}$ bandwidth at the BESSYII (both FWHM).

\begin{tabular}{lcc}
\hline \hline & LCLS & BESSYII \\
\hline Average incident flux $(1 / \mathrm{s})$ & $9.6 \cdot 10^{11}$ & $1 \cdot 10^{11 \mathrm{a}}$ \\
Photons per pulse & $1.6 \cdot 10^{10}$ & 200 \\
Attenuation factor ${ }^{\mathrm{b}}$ & 10 & - \\
Repetition rate $(\mathrm{Hz})$ & 60 & $5 \cdot 10^{8}$ \\
Pulse length $(\mathrm{ps})$ & 0.16 & 60 \\
Spot size on the jet $(\mu \mathrm{m} \times \mu \mathrm{m})$ & $20 \times 300$ & $20 \times 100^{\mathrm{c}}$ \\
Fluence per pulse $\left(\mathrm{mJ} / \mathrm{cm}^{2}\right)$ & 31 & $1.4 \cdot 10^{-6}$ \\
Peak fluence $\left(1 /\left(\mathrm{ps} \times \mu \mathrm{m}^{2}\right)\right)$ & $1.7 \cdot 10^{7}$ & 0.002 \\
Measured count rate $(1 / \mathrm{s})$ & 480 & 10 \\
Estimated count rate $(1 / \mathrm{s})^{\mathrm{d}}$ & 160 & - \\
\hline \hline
\end{tabular}

a The value is reduced by a factor which takes into account the overlap of the x-ray beam with the jet (jet diameter $20 \mu \mathrm{m}$ ).

$\mathrm{b}$ The attenuation factor is given with respect to an unattenuated but monochromatized beam. Compared to the unmonochromatized beam the attenuation is $\sim 300$.

c The horizontal spot size of $20 \mu \mathrm{m}$ corresponds to the diameter of the jet here.

d The estimated LCLS count rate is calculated from the measured count rate at BESSYII taking into account the different average incident flux. The discrepancy between measured and estimated LCLS count rate reflects the uncertainties in the average incident photon fluxes of LCLS and BESSYII.

nels are necessary. Therefore we make no attempt here to generalize our results to other compunds. We note that the threshold for fluence dependent radiation damage depends on the specific sample and it is essential to determine this experimentally in case of a new sample and/or photon beam parameters.

\section{CONCLUSIONS}

We have demonstrated the feasibility of soft x-ray RIXS experiments in liquid phases at FELs with a novel experimental setup combining an x-ray emission spectrometer with an in vacuum liquid jet. Due to very high peak fluences at FELs a new type of radiation damage effect occurs which is not present at synchrotron light sources. This fluence dependent radiation damage effect sets a limit for the maximum incident photon flux possible to use at FELs and thus limits the count rate in RIXS experiments. Compared to the stateof-the-art synchrotron beamline U41-PGM at BESSYII, at least a factor of 40 smaller incident photon fluxes were necessary to use at the LCLS SXR beamline in experiments on pure bulk samples (corresponding to $8 \cdot 10^{6}$ photons $/\left(\mathrm{ps} \times \mu \mathrm{m}^{2}\right)$ threshold fluence of methanol at $160 \mathrm{fs}$ pulse length). For the case of a dilute sample 
we observed a higher threshold for fluence dependent radiation damage. This makes it feasible to perform experiments on dilute samples with practically the same count rate at LCLS as at the beamlines of BESSYII. We also note that the possibility to adjust x-ray pulse length and spot size could make it possible in the future to utilize the full photon flux available at FELs.

The setup can be used for femtosecond time-resolved experiments in a conventional pump-probe scheme provided by the FEL facilities. In addition, the setup can also be used for complementary static experiments at synchrotron facilities. All this makes the setup ideal for novel experiments on molecular dynamics and real-time chemistry in the liquid phase.

\section{ACKNOWLEDGMENTS}

We want to cordially thank Carlo Schmidt and Erzsi Szilagyi for valuable help during the LCLS beamtime, Kerstin Kalus, Annette Pietzsch, Carl-Johan Englund, Markus Agåker, and Conny Såthe for support during the commissioning of the setup, Nils Mårtensson for generously making the RIXS spectrometer available, the LCLS SXR team especially Michael Rowan, Tom Benson, Oleg Krupin, Michael Holmes, the LCLS controls and DAQ team especially Marc Messerschmidt, Bruce Hill, Wilfred Ghonsalves, the LCLS floor coordinators team and the SLAC/MCC operations team for dedication and excellent support, the SLAC/SSRL/LCLS safety especially Ian Evans and Cynthia Patty for efficient cooperation, and Aaron Lindenberg and Erik Nibbering for cooperation during the proposal phase of the LCLS beamtime. We gratefully acknowledge support by the staff of the Helmholtz-Zentrum Berlin for support during the BESSYII beamtimes. S. T. is grateful for financial support by the Advanced Study Group and DFG support through SFB 602 and SFB 755 . W. Q. is thankful for financial support through the SFB 755 , Nanoscale Photonic Imaging. Financial support is given to M. B. by the VolkswagenStiftung. I. R. is thankful for scientific support through the ASG of the MPG at CFEL. W. Z., R. W. H., and K. J. G. acknowledge support from the AMOS program within the Chemical Sciences, Geosciences, and Biosciences Division of the Office of Basic Energy Sciences, Office of Science, U. S. Department of Energy. Portions of this research were carried out on the SXR Instrument at the Linac Coherent Light Source (LCLS), a division of SLAC National Accelerator Laboratory and an Office of Science user facility operated by Stanford University for the U.S. Department of Energy. The SXR Instrument is funded by a consortium whose membership includes the LCLS, Stanford University through the Stanford Institute for Materials Energy Sciences (SIMES), Lawrence Berkeley National Laboratory (LBNL), University of Hamburg through the BMBF priority program FSP 301, and the Center for Free Electron Laser Science (CFEL).

\section{REFERENCES}

${ }^{1}$ A. Nilsson, L. G. M. Petterson, and J. Nørskov, eds., Chemical bonding at surfaces and interfaces (Elsevier Science, 2007).

${ }^{2}$ L. J. P. Ament, M. van Veenendaal, T. P. Devereaux, J. P. Hill, and J. van den Brink, Rev. Mod. Phys. 83, 705 (2011).

${ }^{3}$ A. Föhlisch, M. Nyberg, P. Bennich, L. Triguero, J. Hasselström, O. Karis, L. G. M. Pettersson, and A. Nilsson, J. Chem. Phys. 112, 1946 (2000).

${ }^{4}$ A. Föhlisch, P. Feulner, F. Hennies, A. Fink, D. Menzel, D. Sanchez-Portal, P. M. Echenique, and W. Wurth, Nature 436, 373 (2005).

${ }^{5}$ A. Pietzsch, Y. P. Sun, F. Hennies, Z. Rinkevicius, H. O. Karlsson, T. Schmitt, V. N. Strocov, J. Andersson, B. Kennedy, J. Schlappa, A. Föhlisch, J. E. Rubensson, and F. Gel'mukhanov, Phys. Rev. Lett. 106, 153004 (2011).

${ }^{6}$ M. Beye and A. Föhlisch, Chemical Physics (2012), 10.1016/j.chemphys.2012.03.023.

${ }^{7} \mathrm{Ph}$. Wernet, Phys. Chem. Chem. Phys. 13, 16941 (2011).

${ }^{8}$ M. Beye, Ph. Wernet, C. Shüssler-Langeheine, and A. Föhlisch, J. Electron Spectrosc. Relat. Phenom. (submitted).

${ }^{9}$ N. Huse, H. Cho, K. Hong, L. Jamula, F. M. F. de Groot, T. K. Kim, J. K. McCusker, and R. W. Schönlein, J. Phys. Chem. Lett. 2, 880 (2011).

${ }^{10}$ H. Wen, N. Huse, R. W. Schönlein, and A. M. Lindenberg, J. Chem. Phys. 131, 234505 (2009).

${ }^{11}$ N. Huse, H. Wen, D. Nordlund, E. Szilagyi, D. Daranciang, T. A. Miller, A. Nilsson, R. W. Schönlein, and A. M. Lindenberg, Phys. Chem. Chem. Phys. 11, 3951 (2009).

${ }^{12} \mathrm{Ph}$. Wernet, in 14 th International Conference On X-ray Absorption Fine Structure (XAFS14), Proceedings, Journal of Physics Conference Series, Vol. 190, edited by A. DiCicco and A. Filipponi, IOP (IOP Publishing LTD, Dirac House, Temple Back, Bristol BS1 6be, England, 2009) p. 012055.

${ }^{13}$ G. Gavrila, K. Godehusen, C. Weniger, E. T. J. Nibbering, T. Elsaesser, W. Eberhardt, and P. Wernet, Appl. Phys. A-Mater. Sci. Process. 96, 11 (2009).

${ }^{14} \mathrm{Ph}$. Wernet, G. Gavrila, K. Godehusen, C. Weniger, E. Nibbering, T. Elsaesser, and W. Eberhardt, Appl. Phys. A 92, 511 (2008).

${ }^{15}$ G. Vanko, P. Glatzel, V.-T. Pham, R. Abela, D. Grolimund, C. N. Borca, S. L. Johnson, C. J. Milne, and C. Bressler, Angew. Chem. Int. Ed. 49, 5910 (2010).

${ }^{16}$ M. Beye, F. Sorgenfrei, W. F. Schlotter, W. Wurth, and A. Föhlisch, Proc. Natl. Acad. Sci. U. S. A. 107, 16772 (2010).

${ }^{17}$ K. M. Lange, R. Könnecke, S. Ghadimi, R. Golnak, M. A. Soldatov, K. F. Hodeck, A. Soldatov, and E. F. Aziz, Chem. Phys. 377, 1 (2010).

${ }^{18}$ M. Blum, L. Weinhardt, O. Fuchs, M. Baer, Y. Zhang, M. Weigand, S. Krause, S. Pookpanratana, T. Hofmann, W. Yang, J. D. Denlinger, E. Umbach, and C. Heske, Rev. Sci. Instrum. 80, 123102 (2009).

${ }^{19}$ T. Tokushima, Y. Harada, O. Takahashi, Y. Senba, H. Ohashi, L. G. M. Pettersson, A. Nilsson, and S. Shin, Chem. Phys. Lett. 460, 387 (2008).

${ }^{20}$ J. Guo, Y. Luo, A. Augustsson, J. Rubensson, C. Såthe, H. Ågren, H. Siegbahn, and J. Nordgren, Phys. Rev. Lett. 89, 137402 (2002).

${ }^{21}$ W. F. Schlotter, J. J. Turner, M. Rowen, P. Heimann, M. Holmes, O. Krupin, M. Messerschmidt, S. Moeller, J. Krzywinski, R. Soufli, M. Fernandez-Perea, N. Kelez, S. Lee, R. Coffee, G. Hays, M. Beye, N. Gerken, F. Sorgenfrei, S. Hau-Riege, L. Juha, J. Chalupsky, V. Hajkova, A. P. Mancuso, A. Singer, O. Yefanov, I. A. Vartanyants, G. Cadenazzi, B. Abbey, K. A. Nugent, H. Sinn, J. Luening, S. Schaffert, S. Eisebitt, W. S. Lee, A. Scherz, A. R. Nilsson, and W. Wurth, Rev. Sci. Instrum. 83, 043107 (2012).

${ }^{22}$ O. Krupin, M. Trigo, W. F. Schlotter, M. Beye, F. Sorgenfrei, J. J. Turner, D. A. Reis, N. Gerken, S. Lee, W. S. Lee, G. Hays, Y. Acremann, B. Abbey, R. Coffee, M. Messerschmidt, 
S. P. Hau-Riege, G. Lapertot, J. Luening, P. Heimann, R. Soufli, M. Fernandez-Perea, M. Rowen, M. Holmes, S. L. Molodtsov, A. Foehlisch, and W. Wurth, Optics Express 20, 11396 (2012).

${ }^{23}$ J. Hallmann, S. Grübel, I. Rajkovic, W. Quevedo, G. Busse, M. Scholz, R. More, M. Petri, and S. Techert, J. Phys. B-at. Mol. Opt. 43, 194009 (2010).

${ }^{24}$ I. Rajkovic, G. Busse, J. Hallmann, R. More, M. Petri, W. Quevedo, F. Krasniqi, A. Rudenko, T. Tschentscher, N. Stojanovic, S. Düsterer, R. Treusch, M. Tolkiehn, and S. Techert, Phys. Rev. Lett. 104, 125503 (2010).

${ }^{25}$ I. Rajkovic, J. Hallmann, S. Grübel, R. More, W. Quevedo, M. Petri, and S. Techert, Rev. Sci. Instrum. 81, 045105 (2010).

${ }^{26}$ A. Charvat, E. Lugovoj, M. Faubel, and B. Abel, Rev. Sci. Instrum. 75, 1209 (2004).

${ }^{27}$ M. Faubel, B. Steiner, and J. P. Toennies, J. Chem. Phys. 106, 9013 (1997).

${ }^{28}$ M. Faubel, K. R. Siefermann, Y. Liu, and B. Abel, Acc. Chem. Res. 45, 120 (2012).

${ }^{29}$ J. Nordgren, G. Bray, S. Cramm, R. Nyholm, J.-E. Rubensson, and N. Wassdahl, Rev. Sci. Instrum. 60, 1690 (1989).
${ }^{30}$ J. Nordgren and J. Guo, J. Electron Spectrosc. Relat. Phenom. 110, 1 (2000).

${ }^{31}$ F. Schäfers and M. Krumrey, "REFLEC - a program to calculate VUV and soft x-ray optical elements and synchrotron radiation beamlines," Tech. Rep. 1-17 (BESSY TB 201, 1996).

${ }^{32}$ F. Schäfers, "RAY - the BESSY raytrace program to calculate synchrotron radiation beamlines," Tech. Rep. 1-37 (BESSY TB 202, 1996).

${ }^{33}$ F. Schäfers, "Springer series in modern optical sciences: Modern development in X-ray and neutron optics," (Springer, 2008) Chap. RAY - The BESSY raytrace program, pp. 9-41.

${ }^{34}$ B. Henke, E. Gullikson, and J. Davis, At. Data and Nuc. Data Tables 54, 18 (1993).

${ }^{35}$ H. G. Beutler, J. Opt. Soc. Am. 35, 311 (1945).

${ }^{36}$ T. Namioka, J. Opt. Soc. Am. 49, 446 (1959).

${ }^{37}$ J. Guo, Y. Luo, A. Augustsson, S. Kashtanov, J. Rubensson, D. Shuh, H. Ågren, and J. Nordgren, Phys. Rev. Lett. 91, 157401 (2003).

${ }^{38}$ S. Kashtanov, A. Augustson, J.-E. Rubensson, J. Nordgren, H. Ågren, J.-H. Guo, and Y. Luo, Phys. Rev. B 71, 104205 (2005). 